\title{
DISCUSSION
}

$P$. A. Sturrock. It may be possible to obtain a unified treatment of the effect of convection on the mean profile and the effect of the mean profile on convection by using techniques similar to those of the quasi-linear theory of instabilities in plasmas which gives a unified treatment of the effect of electrostatic waves on the electron distribution function and the influence of the distribution function on electrostatic waves.

M. Schwarzschild. Dr Böhm's recent theoretical work on the convective modes in the solar photosphere has induced us to reconsider our Stratoscope data for the power spectrum of the solar granulation. Under the most pessimistic assumptions for the instrumental profile of Stratoscope I, permitted by the observed solar limb profile, we now obtain a granulation power spectrum which could reach substantially farther to smaller wave numbers than our old spectrum did. This helps to reduce the discrepancy between theory and observation mentioned by Dr Spiegel.

A. Cook. Your description is in terms of an exponential increase with time. But this cannot go on for ever, so your description is appropriate to the onset of convection but not to a 'quasistable' state. What difference will this make to the spectrum of velocities? Would it lead to a more peaked spectrum?

E. A. Spiegel. The description refers in principle, not to a stability problem, but to fully turbulent convection. In this case, there are two effects of nonlinearity: one is the distortion of the average state of the atmosphere by convective heat transfer; the other is the coupling between fluctuating modes. The first effect is included in the calculation of growth rates since the linearization is made about the turbulent atmosphere whose structure is found approximately from mixinglength calculations. These growth rates measure the driving force on a mode in the mean state of the turbulent fluid. The growth rate thus tells us which modes are driven and which are not. But as you have recognized we cannot learn the limiting amplitude without including the coupling through the fluctuating nonlinear terms, hence we cannot predict the shape of the spectrum globally. I wanted merely to point out, that the spectrum seen at the top of the convective zone is greatly influenced by local conditions and may appear peaked, even though the spectrum in the interior is rather different. So even without the fluctuating interactions, the growth rate alone does not tell the whole story-we must also know the nature of the normal modes.

\section{OSCILLATORY AND WAVE MOTION IN STELLAR ATMOSPHERES}

\author{
E. Schatzman \\ (Institut d'Astrophysique, Paris)
}

We shall try, in the following, to consider only the basic problems of wave motions in stellar atmospheres. Not considering the impossibility, in such a lecture, of any kind of mathematical treatment, we shall keep in mind the idea that the physics of the problem is the most important matter to consider.

First, we have to consider the structure of the atmosphere in which we want to study the wave propagation. The actual atmospheres are very far from the idealized models which have been used consistently for many years. However, several of these idealized models are extremely 
useful, as they show a certain number of properties which seem to be essential for our understanding of real atmospheres.

It is well known that an isothermal atmosphere is a dispersive medium, and that for a given direction of propagation, there is an interval of frequencies for which no propagation is possible. For the higher frequencies, the waves are of the compressive type, whereas for the lower frequencies, the waves are of the gravitational type. These properties have been recently thoroughly studied by Moore and Spiegel, and we can understand how the oscillations which have been produced in the deeper layers of the atmosphere, in the convective zone, can reach the upper layers and provide the flux of mechanical energy necessary for the coronal heating.

However, the consideration of an isothermal atmosphere hides completely one of the basic properties of stellar atmosphere. In an isothermal atmosphere, the waves are free-waves; in other words, the only characteristic length associated with the atmosphere is the scale height, and the geometrical structure of the atmosphere does not determine the wave structure.

The atmosphere of the Sun is not isothermal. It is characterized by a temperature minimum. In other words, the dispersive properties of the atmosphere depend on height and this introduces another characteristic length. For example, a low frequency wave would correspond to a progressive wave in the upper and in the lower part of the atmosphere, and to a standing wave in the intermediate region of temperature minimum. Such a situation, where the velocity of propagation is real in two regions and imaginary in the intermediate region is fairly well known and has been investigated since many years. Astrophysicists are only now rediscovering, in a special context, what has been known to hydrodynamicists and to quantum physicists. What is characteristic of such a situation is that waves are reflected and transmitted. Interferences can take place or, in other words, the system shows eigen-properties, with eigen-values.

We can consider the eigen-properties in two different ways. If we consider an incident progressive wave coming from below, it is reflected by the non-propagating region, and transmitted with a different amplitude in the upper region, as a progressive wave. The relation between the amplitudes of the transmitted and of the incident waves depend of the frequency and show a finite or an infinite number of poles. Most generally, these poles are given by complex values of the frequencies. Physically speaking the existence of such complex values can be understood in the following way. When the frequency takes one of the eigen-values, the amplitude of the incident progressive waves vanishes. In other words, the region where no progressive wave can take place oscillates, and radiates acoustically both in the upper and in the lower regions. The losses of energy due to the acoustic radiation (either due to compression or to gravitational waves) are necessarily accompanied by a decay of the amplitude of the oscillation, and therefore, the frequency is complex, the imaginary part of the frequency being positive.

It is naturally possible to look for the complex eigen-values of the frequency for different models of the atmosphere. Bahng and Schwarzschild have considered a few models of the solar atmosphere, chromosphere and corona, with special choices in parameters, such that the problem could be mathematically tractable. Other examples have been considered by the author of this report, and the details will appear in a separate publication. However when looking for eigen values, one misses completely the important relation between the amplitude of the incident and transmitted wave. Schmidt and Zirker have considered the transmission by an atmosphere of the wave produced by a piston which suddenly begins to move upwards at time $t=0$. Their result reflects essentially the dispersive properties of the atmosphere, but misses completely a very important point. The eigen-frequencies are preferentially excited by the motions in the lower layers of the atmosphere, and the spectrum of the transmitted oscillation reflects both the properties of the impressed force and the filtering properties of the atmosphere. Following Bahng and Schwarzschild, the author of the report has considered two atmospheric models, with a special choice of the parameters, such that the models are mathe- 
matically tractable. For an impressed force which is a step function, the transmitted wave bears some similarity with the oscillations observed by Evans and Michard, with a fast damping, and only a few visible oscillations. However, the numerical value of the apparent period of oscillation is not in quantitative agreement with the observed value of 285 seconds. The difference can well be due to the choice of the parameters, which was made essentially with a purpose of mathematical simplicity. The result is encouraging, and it would certainly be worth to study in detail the transmission properties of a model closer to reality.

In the following, we shall mention a few problems which are waiting for their solution.

The waves which have been generated in the convective zone propagate with a growing amplitude in the upper atmosphere. The exact way in which the waves become shock waves has not been investigated in detail, and the theory of the dissipative processes has still to be improved. Noyes and Leighton have studied the effects of radiation on the phase lag problem; their result is of the greatest importance, as it shows the possible explanation of the change with height of the phase lag between temperature and velocity. It would certainly be most useful to study the transmission properties of an atmosphere, including the radiation effect.

Another aspect of the problem results from the difference in propagation properties and rate of dissipation as a function of the magnetic field intensity. As suggested by Shklovsky and studied by Parker, the large scale motions in the convective zone result in the production of inhomogeneities of the magnetic field. Along the chromospheric network, the field seems to be stronger. It is quite likely that the differences in the flux of mechanical energy and in the rate of dissipation could account for the optical aspect of the chromospheric network. The structure of the spicules which seem to appear in bushes placed also on the chromospheric network, seem to be related to the same problem. However, it would be necessary to consider the propagation problem and the heat balance problem in a matter having the peculiar thermodynamic properties of the chromosphere and lower corona. Work in that direction is in progress at the Institut d'Astrophysique.

The problem of wave propagation is also related to the old problem of heating of the solar atmosphere and corona. Spherically symmetrical models have certainly provided all what they could give. The corona is certainly highly heterogeneous and regions of different magnetic field intensity have certainly a different thermal balance. Two problems arise in this connection:

I. One is the coupling of the different modes of propagation. Compression waves produced in the convection zone and reaching the lower chromosphere, propagate in regions of non uniform density and non uniform magnetic field. Through these gradients compression waves can generate Alfvén waves which can propagate, along the magnetic lines of force, in the corona. To solve the exact problem of the coupling is extremely difficult. Frisch has approached the solution in the case of twisted lines of force in a stratified medium. But the problem is still awaiting its solution and is basic to the understanding of the structure of the corona.

2. The second problem concerns the rate of dissipation of the waves in presence of a magnetic field. Various approaches have been given to the problem, but it should be worth remembering that the exact evaluation of the damping constant of a wave in a plasma, in presence of a magnetic field, is a very intricate problem of kinetic theory of gases. Work in that direction is also in progress at the Institut d'Astrophysique and has been undertaken in connection with the question of coronal heating.

The present solution of the studies of oscillatory and wave motions in stellar atmospheres is encouraging. Many problems begin to be understood, many problems are awaiting their solution. It is always a pleasure to find oneself with some work to do. 


\section{REFERENCES}

Alfvén, H. Mon. Not. R. astr. Soc., 107, 211 , 1947.

Bahng, J., Schwarzschild, M. Astrophys. F., 137, 901, 1963.

Bazer, J., Huley, J. $\quad$ F. Geophys. Res., 68, 147, 1963.

Budden, K. G. Radio-waves in the ionosphere, Cambridge University Press, I961.

Dungey, J. W. Cosmic electrodynamics, Cambridge University Press, 1958.

Eckart, C. Hydrodynamics of oceans and atmospheres, Pergamon Press, 1960.

Ferraro, V. C. A. Astrophys. F., rr9, 393, I954.

Frisch, U. Ann. Astrophys., 27, 224, 1964.

Grad, H. Rev. mod. Phys., 32, 830, 1960.

Heading, J. An introduction to phase integral methods, Methuen \& Co, London, r 962.

Kahn, F. D. Astrophys. F., 135, 547, 1962.

Keller, J. B. F. appl. Phys., 25, 938, I954.

Kogure, J., Osaki, I. Publ. astr. Soc. Fapan, 14, 254, 1962.

Lamb, H. Hydrodynamics, Cambridge University Press, 1945.

Lehnert, B. Nuovo Cim. Suppl. ser. 10, 13, 59, 1959.

Lighthill, M. J. Phil. Trans. R. Soc. A., 252, 397, 1960.

Moore, D. W., Spiegel, E. A. Astrophys. F., 139, 48, 1964.

Noyes, R. W., Leighton, R. B. Astrophys. F., 138, 631, 1963.

Osterbrock, D. E. Astrophys. F., 134, 347, $196 \mathrm{r}$.

Parker, E. N. Astrophys. F., 138, 226, 1961.

Piddington, J. H. Mon. Not. R. astr. Soc., 116, 314 , 1956.

Plumpton, C. Astrophys. F., 129, 752, 1959.

Plumpton, C., Ferraro, V. C. A. Astrophys. F., 127, 459, 1958.

Poisson, S. D. F. Ecole Polytechn., 7, 334, I807.

Raylighn, J. W. S. Phil. Mag., 29, 173, 1890.

Saito, M. Publ. astr. Soc. Japan, 16, 179, 1964.

Schatzman, E. Ann. Astrophys., 12, 203, r949; Bull. Acad.r. Belg. Cl. Sci., 40, 139, 1954;

Nuovo Cim. Suppl., 22, 1047, 1961; Festschrift f. S. Rossland. Astrophysica Norvegica, 9, 283,1965 .

Schmidt, H. U., Zirker, J. B. Astrophys. F., 138, 1310, 1963.

Spiegel, E. A. Astrophys. $\mathscr{F}$., 126, 202, 1947.

Spiegel, E. A. Astrophys. \%., 139, 959, 1964.

Walén, C. Ark. Mat. Astr. Fys., 30A, no. 15, 1944.

Weymann, R., Howard, R. Astrophys. F., 128, 142, 1958.

Whitham, G. B. F. Fluid Mech., 4, 337, 1958.

Whitney, C. A. Astrophys. $\mathcal{F} .$, 138, 537, 1963.

Whittaker, W. A. Astrophys. F., r37, 9r4, r963.

\section{DISCUSSION}

R. F. Christy. I would like to add a few comments relevant to the hydrogen zone convection in cool super-giants, particularly F, G, and $\mathrm{K}$. This is the region, I believe, where convection takes on its most violent, compressive behaviour and an understanding of this kind of behaviour may also help us understand convection in the hydrogen zone in other stars.

In the calculations I have carried out on RR Lyrae and Cepheid models, it has become apparent that there can be a strong coupling of compressive modes and heat flow in the region $20000^{\circ} \mathrm{K}$. In particular there has appeared a form of thermal instability in this region, superimposed on the pulsation motion. It seems very likely from these investigations that this region can be the seat of a spontaneous generation of compressive waves which then travel out to the surface with rapid increase in amplitude and carry some of the energy into the photosphere. These waves, in my work, are closely related to the bumps on the falling branch of the luminosity 
curve. They can well involve supersonic velocities in the atmosphere and may be the explanation of the so-called 'supersonic turbulence' reported observationally in super-giants. In my work they have appeared with fairly characteristic periods of about one-eighth of the pulsation period. It would be of interest if such periodicity could be found observationally in supergiants. I would like to add that the techniques which have been successfully applied to calculating the non-linear time dependent motions in Chheids and RR Lyrae stars, can also be applied to a detailed non-linear study of one-dimensional instabilities in convection zones such as the spontaneous generation of radial sound waves.

$F$. Edmonds. The temporal power spectra from lines formed at different depths shown as a slide by Michard shows that the fractional contribution of the oscillatory component of velocity fluctuations is 0.55 at $\tau \simeq 0.5$, and that though this contribution is decreasing with increasing depths, it probably will still be important in the convection zone where $\tau>\mathrm{I}$.

\title{
5. OUTER CONVECTION ZONES IN STARS OF DIFFERENT SPECTRAL TYPES AND LUMINOSITIES
}

\author{
E. Böhm-Vitense \\ (University of Heidelberg)
}

It seems to me that the general situation in the theory of convection zones has not changed very much during the last years. There are two different attitudes towards this problem. Some people try very hard to develop a correct theory of turbulent convection in a medium where the density and the instability changes by orders of magnitude within the unstable layer. Though our understanding of the hydrodynamics has improved quite a bit and we can even draw some nice pictures of convection as we have seen from the very interesting talk of Dr Spiegel this morning, it seems to me that we are still far from having a theory applicable to compute the velocities and the convective energy transport in real stars.

Then there is the other groups of astronomers using the only applicable theory of convection developed so far, namely the so-called mxing length theory with its well-known approximations and uncertainties, trying to find out at least some properties of the convection zones. There have been attempts to improve the mixing length theory (for instance Spiegel ( $x$ )) but its major uncertainty remains, namely the size of the characteristic length.

So what can we say about the outer convection zones under these circumstances?

I would like to concentrate on four points:

I. Origin of instability against convection.

2. Upper boundary of convection zones.

3. Amount of energy transported by convection.

4. Velocities of convection currents to be expected.

I. The well-known criterion for instability against convection is

$$
\nabla_{\mathrm{r}}=\left(\frac{\mathrm{d} \ln T}{\mathrm{~d} \ln P_{\mathrm{g}}}\right)_{\text {radiative equilibrium }}>\left(\frac{\mathrm{d} \ln T}{\mathrm{~d} \ln P_{\mathrm{g}}}\right)_{\text {adiabatic }}=\nabla_{\mathrm{a}},
$$

here $\nabla_{\mathrm{a}}<0.4$ where the sign $<$ applies if an abundant element ionizes. We then have to compute $\nabla_{\mathrm{r}}$. With a temperature-distribution

$$
T^{4}=\mathrm{T}_{e f f}^{4}(\bar{\tau}+q(\bar{\tau}))
$$

